\title{
Molecular characterization of extended-spectrum $\beta$-lactamase-producing Escherichia coli isolates from chickens in Henan Province, China
}

\begin{abstract}
Correspondence
Gong-Zheng Hu

yaolilab@163.com
\end{abstract}

Received 4 May 2009

Accepted 30 June 2009

\author{
Li Yuan, ${ }^{1}$ Jian-Hua Liu, ${ }^{1}$ Gong-Zheng Hu, ${ }^{1}$ Yu-Shan Pan, ${ }^{1}$ Zhi-Ming Liu, ${ }^{2}$ \\ Juan $\mathrm{Mo}^{1}$ and Yong-Jun Wei ${ }^{1}$
}

${ }^{1}$ Department of Pharmacology and Toxicology, College of Animal Husbandry and Veterinary
Science, Henan Agricultural University, Zhengzhou 450002, PR China

${ }^{2}$ Henan Bureau of Animal Husbandry, Zhengzhou 450002, PR China

\section{INTRODUCTION}

Extended-spectrum $\beta$-lactamases (ESBLs) are rapidly spreading worldwide (Tenover et al., 1999) and presently comprise over 500 variants (http://www.lahey.org/Studies), which are frequently encountered among human and animal clinical Enterobacteriaceae isolates (Bradford, 2001). A typical characteristic of ESBLs is their ability to hydrolyse oxyimino-cephalosporins, which can be inhibited by $\beta$ lactamase inhibitors (Paterson \& Bonomo, 2005). ESBLs, particularly TEM, SHV and CTX-M enzymes, exhibit a high degree of diversity (Bonnet, 2004; Livermore et al., 2007). ESBL genes are usually carried by plasmids, facilitating their spread among Gram-negative bacilli. Several surveillance studies have revealed a relatively high

Abbreviations: ESBL, extended-spectrum $\beta$-lactamase; MDR, multidrug resistant.

The GenBank/EMBL/DDBJ accession numbers for the sequences of the majority of the $\beta$-lactamases from the 31 isolates in this study are FJ405191-FJ405214 and FJ812104-FJ812110. prevalence of ESBL-producing organisms in the AsiaPacific area (Hirakata et al., 2005; Wang et al., 2005). In particular, the CTX-M family is believed to be dominant in Asia, as it has appeared or caused outbreaks in many countries (Bonnet, 2004; Munday et al., 2004; Ryoo et al., 2005; Kiratisin et al., 2008; Xiao et al., 2008).

Since 1994, ESBL-producing bacteria have become widely disseminated in the People's Republic of China and their molecular characterization has focused mainly on human clinical isolates (Cheng \& Chen, 1994; Chanawong et al., 2002; Wang et al., 2005; Xiao et al., 2008; Liu et al., 2009). Several studies worldwide have studied the epidemiology and molecular characterization of ESBL-producing animal clinical isolates (Batchelor et al., 2005; Briñas et al., 2005; Riaño et al., 2006; Smet et al., 2008), whereas similar reports in China have been rare (Duan et al., 2006; Liu et al., 2007; Hu et al., 2008). Here, we report the results of the first extensive phenotypic and molecular studies of ESBL-producing Escherichia coli isolated from 14 different chicken farms in Henan Province, China. The aim of the 
study was to determine the ESBL frequency in clinical E. coli isolates from chickens and to reveal basic aspects of their molecular epidemiology.

\section{METHODS}

Bacterial strains. A total of 51 non-replicate isolates were collected between December 2007 and August 2008 from 14 chicken farms in Henan Province. All samples were obtained aseptically from liver swabs as soon as the sick chickens died, and the organisms were seeded immediately on MacConkey agar. Each isolate was collected from a single chicken. The isolates were identified as E. coli using the Vitek-32 system and showed resistance to ampicillin according to the Clinical and Laboratory Standards Institute (CLSI) broth microdiffusion method (CLSI, 2007). The azide-resistant E. coli DH5 $\alpha$ was used as a recipient strain for conjugative transfer. E. coli ATCC 25922 (Beijing Ordinary Microbiology Strain Store Center), E. coli CF1124 (producing TEM-24; Peking University Health Science Center), E. coli ZM-7 (CTX-M-type, GenBank accession no. DQ849332, identified by our laboratory) and Klebsiella pneumoniae ATCC 700603 (producing SHV-18; Peking University Health Science Center) were used as reference strains.

Identification of ESBL producers. ESBL-producing isolates were screened by a phenotypic confirmatory test using cefotaxime, ceftazidime, cefotaxime/clavulanate $(2: 1)$ and ceftazidime/clavulanate $(2: 1)$ according to CLSI recommendations (CLSI, 2007). All ESBL-producing isolates were collected for further investigation.

Antimicrobial susceptibility test. The antimicrobial susceptibilities of all ESBL-producing clinical isolates were determined at the same time using the CLSI broth microdilution method (CLSI, 2007). The antimicrobial agents tested were: ceftiofur, ceftiofur/sulbactam $(2: 1)$, amoxicillin/clavulanate $(2: 1)$, cefoperazone, cefoperazone/tazobactam (2:1), ceftriaxone, cefepime, amikacin, gentamicin, florfenicol, doxycycline, sulfamethoxazole/trimethoprim $(5: 1)$ and enrofloxacin. Isolates shown to be resistant to at least three different classes of agent were classified as multidrug resistant (MDR) (Kiratisin et al., 2008).

Characterization of genes encoding $\boldsymbol{\beta}$-lactamases. As most ESBL genes have been shown to be plasmid-borne, plasmid DNA was extracted and purified using a TaKaRa MiniBEST Plasmid Purification kit. PCR amplification of $b l a_{\mathrm{TEM}}, b l a_{\mathrm{SHV}}$ and $b l a_{\mathrm{CTX}-\mathrm{M}}$ was performed with TaKaRa Ex Taq DNA polymerase using the primers listed in Table 1, as described previously (Hu et al., 2006). All PCR amplicons were verified by gel electrophoresis on a $1.0 \%(\mathrm{w} / \mathrm{v})$ agarose gel and stained with ethidium bromide $\left(0.5 \mu \mathrm{g} \mathrm{ml}^{-1}\right)$. PCR amplicons were purified, subsequently ligated into the pGEM-T Easy vector (Promega) and then sequenced. Sequencing analyses were performed online using BLAST (www.ncbi.nlm.nih.gov/BLAST/).

Conjugation. Conjugation experiments were carried out using E. coli $\mathrm{DH} 5 \alpha$. Transconjugants were selected on tryptic soy agar containing
$200 \mu \mathrm{g}$ sodium azide $\mathrm{ml}^{-1}$ and $50 \mu \mathrm{g}$ cefazolin $\mathrm{ml}^{-1}$ and their $\beta$ lactamase genes were confirmed by PCR as described above.

\section{RESULTS AND DISCUSSION}

\section{E. coli isolates harbouring ESBLs}

With the extensive use in poultry of $\beta$-lactam antibiotics such as amoxicillin and cephalosporins, especially extended-spectrum cephalosporins, ESBL-mediated resistance in Gram-negative bacilli has become increasingly critical, and therapeutic options for such infections are becoming limited ( $\mathrm{Hu}$ et al., 2005; Pfaller \& Segreti, 2006). It is important to detect ESBL producers in order to know the ESBL prevalence in animal-associated bacteria and to limit the spread of these MDR organisms in veterinary settings (Ramphal \& Ambrose, 2006; Liu et al., 2007). In the present study, $60.8 \%(31 / 51)$ of the isolates tested were ESBL-producing E. coli. We showed previously that only $12.9 \%(4 / 31)$ of clinical Enterobacteriaceae isolates were ESBL producers (Hu et al., 2006). Another survey (Liu et al., 2007), in 2007, showed that the prevalence of ESBLproducing animal-associated bacteria in China was $30 \%$ $(15 / 50)$. These results indicate that the incidence of ESBLs in bacteria isolated from animals in China is increasing.

\section{Susceptibility of ESBL-producing $E$. coli to antimicrobial agents}

The $\mathrm{MIC}_{50} / \mathrm{MIC}_{90}$ values of ceftiofur, ceftriaxone, cefoperazone, ceftiofur/sulbactam, amoxicillin/clavulanate, cefoperazone/tazobactam and cefepime against the $31 \mathrm{ESBL}-$ producing $E$. coli isolates were 40/160, 32/128, 64/128, 5/10, $10 / 10,4 / 8$ and $2 / 4 \mu \mathrm{g} \mathrm{ml}^{-1}$, respectively (Table 2). All isolates demonstrated high MICs for extended-spectrum cephalosporins whilst remaining susceptible to $\beta$-lactam $/ \beta$ lactamase inhibitor combinations and cefepime. It is worth noting that the majority of isolates were also resistant to non- $\beta$-lactam agents, especially gentamicin (87.1\%), amikacin $(80.6 \%)$, enrofloxacin $(80.6 \%)$ and sulfamethoxazole/trimethoprim (90.3\%). In addition, $80.6 \%(25 / 31)$ of the ESBL-producing strains expressed an MDR phenotype, suggesting that ESBL-producing isolates have probably acquired additional resistance genes. In order to gain a better understanding of their genetic relationships, further

Table 1. Primers used for PCR amplification of bla genes

\begin{tabular}{|lclcc|}
\hline Target & Primer name & \multicolumn{1}{c|}{ Primer sequence $\left(\mathbf{5}^{\prime} \rightarrow \mathbf{3}^{\prime}\right)$} & Product size $(\mathbf{b p})$ & GenBank accession no. \\
\hline$b l a_{\text {TEM }}$ & TEM-F & GGGGATGAGTATTCAACATTTCC & 861 & AF332513 \\
& TEM-R & GGGCAGTTACCAATGCTTAATCA & & AY036620 \\
& SHV-F & GGTTATGCGTTATATTCGCCTGTG & 861 & AF252622 \\
$b l a_{\text {CTX-M }}$ & SHV-R & TTAGCGTTGCCAGTGCTCGATCA & & \\
& CTX-F & GGGCTGAGATGGTGACAAAGAG & 905 & \\
\hline
\end{tabular}


Table 2. Susceptibilities of ESBL-producing $E$. coli $(n=31)$ to various antimicrobial agents

\begin{tabular}{|c|c|c|c|c|}
\hline \multirow[t]{2}{*}{ Antimicrobial agent(s) } & \multicolumn{3}{|c|}{$\operatorname{MIC}\left(\mu \mathrm{g} \mathrm{ml}^{-1}\right)$} & \multirow[t]{2}{*}{ Resistance (\%) } \\
\hline & Range & $\mathrm{MIC}_{50}$ & $\mathrm{MIC}_{90}$ & \\
\hline Ceftiofur & $0.63-160$ & 40 & 160 & 87.1 \\
\hline Ceftiofur/sulbactam & $0.63 / 0.32-20 / 10$ & 5 & 10 & 0 \\
\hline Amoxicillin/clavulanate & $5 / 2.5-20 / 10$ & 10 & 10 & 0 \\
\hline Cefoperazone & $1-128$ & 64 & 128 & 74.2 \\
\hline Cefoperazone/tazobactam & $0.5 / 0.25-16 / 8$ & 4 & 8 & 0 \\
\hline Ceftriaxone & $0.5-256$ & 32 & 128 & 58.1 \\
\hline Cefepime & $0.5-4$ & 2 & 4 & 0 \\
\hline Gentamicin & $0.63->640$ & 160 & 640 & 87.1 \\
\hline Amikacin & $0.63->640$ & 640 & $>640$ & 80.6 \\
\hline Florfenicol & $0.63-160$ & 5 & 10 & 19.4 \\
\hline Doxycycline & $0.63-80$ & 5 & 40 & 32.3 \\
\hline Sulfamethoxazole/trimethoprim & $16 / 3.2-640 / 128$ & $>640$ & $>640$ & 90.3 \\
\hline Enrofloxacin & $0.63-160$ & 5 & 10 & 80.6 \\
\hline
\end{tabular}

studies are needed to characterize the plasmids on which bla genes and other resistance genes are located.

\section{Molecular characterization of ESBL genes}

Twenty-nine of the 31 ESBL phenotype-positive E. coli isolates (93.5\%) carried bla genes (Table 3). Two isolates did not contain $b l a_{\mathrm{TEM}}, b l a_{\mathrm{SHV}}$ or $b l a_{\mathrm{CTX}-\mathrm{M}}$; further studies will be needed to look for other ESBL genes in these isolates. Among the 29 isolates, $b l a_{\text {CTX-M }}$ was detected in 15 $(51.7 \%)$, and $23(79.3 \%)$ harboured $b l a_{\text {TEM }}$ genes. Nine of the 29 isolates $(31.0 \%)$ carried two bla genes $\left(b a_{\mathrm{TEM}}\right.$ and $\left.b l a_{\mathrm{CTX}-\mathrm{M}}\right)$. None of the isolates contained the $b l a_{\mathrm{SHV}}$ gene. These findings demonstrate that $b l a_{\mathrm{TEM}}$ and $b l a_{\mathrm{CTX}-\mathrm{M}}$ genes are the two dominant types in ESBL-producing chicken isolates and that the CTX-M-type $\beta$-lactamase is playing an increasing role in antibiotic resistance in China. Based on a conjugation assay for selected isolates, all bla genes detected were successfully transferred to transconjugants, suggesting that these genes were plasmid-borne.

One isolate carried $b l a_{\mathrm{TEM}-57}$; the remaining $b l a_{\mathrm{TEM}}$ isolates carried $b l a_{\text {TEM-1 }}$ with one silent nucleotide base variation (T18C) compared with GenBank accession no. AY293072 (TEM-1 $\beta$-lactamase). This indicated that TEM-1 is the

Table 3. Molecular characterization of bla genes among ESBL-producing $E$. coli isolates $(n=31)$

\begin{tabular}{|lc|}
\hline bla gene & No. of isolates \\
\hline TEM-1 & 13 \\
TEM-57 & 1 \\
CTX-M-65 & 6 \\
TEM-1 and CTX-M-14 & 1 \\
TEM-1 and CTX-M-24 & 3 \\
TEM-1 and CTX-M-65 & 5 \\
Non-TEM, non-SHV and non-CTX-M & 2 \\
\hline
\end{tabular}

most common $\beta$-lactamase among $E$. coli isolates from chickens in China. TEM-1, the most prevalent bla-encoded enzyme in human clinical isolates worldwide (Ryoo et al., 2005; Carattoli et al., 2008; Kiratisin et al., 2008; Smet et al., 2008), is not classified as an ESBL. However, several TEM-1 derivatives confer ESBL properties (Paterson \& Bonomo, 2005). In our study, the 13 isolates carrying $b l a_{\mathrm{TEM}-1}$, in the absence of other bla genes, exhibited resistance to thirdgeneration cephalosporins, including ceftiofur, ceftriaxone and cefoperazone. The $\mathrm{MIC}_{50} / \mathrm{MIC}_{90}$ values of these drugs were $40 / 80,32 / 64$ and $64 / 64 \mu \mathrm{g} \mathrm{ml}^{-1}$, respectively. The results suggest that these 13 isolates may carry other ESBL genes that this study did not evaluate, for example OXAtype ESBLs (Paterson \& Bonomo, 2005). Compared with TEM-1, TEM-57 contains a Gly $\rightarrow$ Asp substitution at position 92 (Ambler numbering), which was first described in Proteus mirabilis in 1999 (Bonnet et al., 1999). However, we believe that this is the first study worldwide to report TEM-57 in E. coli and the first $b l_{a_{\mathrm{TEM}}-57}$ sequence deposited in GenBank (accession no. FJ405211). Studies are needed to characterize this enzyme further.

The CTX-M family, first described in 2002 in China (Chanawong et al., 2002) and known to be the most dominant non-TEM, non-SHV ESBLs among members of the Enterobacteriaceae, is recognized as a rapidly growing family of ESBLs in Europe and Asia (Bonnet, 2004; Livermore et al., 2007), with geographical variation in the prevalence of CTX-M cluster groups. In Asia, the CTX-M-9 cluster was found to be the most common (Cantón \& Coque, 2006; Ho et al., 2008; Kiratisin et al., 2008; Song et al., 2009), whilst in Europe the CTX-M-1 cluster was found to predominate (Woodford et al., 2004). The current study showed that, among 29 ESBL-producing E. coli

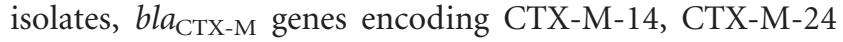
and CTX-M-65 were found in 3.5, 10.3 and $37.9 \%$ of the isolates, respectively, suggesting that there might be wide dissemination of different CTX-M-type $\beta$-lactamases in chicken isolates in China. Based on amino acid sequence 
similarities, CTX-M-14, CTX-M-24 and CTX-M-65 are classified in the CTX-M-9 subgroup. CTX-M-14 was found to be the predominant $\beta$-lactamase in human clinical ESBL-containing E. coli isolates in China (Munday et al., 2004; Liu et al., 2009) and its sequence was determined from China in 2000 (GenBank accession no. AF252622). The nucleotide change in the protein coding region (A823C) between CTX-M-24 and CTX-M-14 leads to the amino acid alteration Ser275Arg, but the resistant profiles are similar. CTX-M-65, another variant of CTX-M-14, was first detected in E. coli isolates from Germany in 2007 (GenBank accession no. EF418608). In 2008, CTX-M-65producing isolates were identified in China (GenBank accession no. EF394372) and America (Doi et al., 2008), but, to our knowledge, this is the first study to report CTXM-65 in animal isolates, and the CTX-M-65 prevalence $(37.9 \%)$ was remarkably high. The amino acid sequence of CTX-M-65 was found to differ from that of CTX-M-14 by the substitutions of Ala80Val and Ser275Arg. Antimicrobial susceptibility tests demonstrated that the CTX-M-65 isolates exhibited a higher level of resistance (from two- to eightfold) to ceftriaxone, ceftiofur, cefoperazone, gentamicin, amikacin and florfenicol compared with CTX-M-14 and CTX-M-24 isolates, i.e. the CTX-M-65 isolates were more prone to exhibit an MDR phenotype. Therefore, these surveys indicated increasing trends not only in the prevalence of CTX-M-65 ESBLs but also in MDR phenotypes in China.

In summary, we have reported the first extensive study of the prevalence and molecular characterization of ESBLproducing E. coli isolated from chickens in Henan Province, China. This study clearly indicated that TEM-1 and CTX-M-65 are widespread in this region. Most ESBL producers were resistant to extended-spectrum cephalosporins and exhibited a high rate of the MDR phenotype.

\section{ACKNOWLEDGEMENTS}

This work was supported by a grant from the National Natural Science Foundation of China (no. 30771624).

\section{REFERENCES}

Batchelor, M., Threlfall, E. J. \& Liebana, E. (2005). Cephalosporin resistance among animal-associated Enterobacteria: a current perspective. Expert Rev Anti Infect Ther 3, 403-417.

Bonnet, R. (2004). Growing group of extended-spectrum $\beta$ lactamases: the CTX-M enzymes. Antimicrob Agents Chemother 48, $1-14$.

Bonnet, R., De Champs, C., Sirot, D., Chanal, C., Labia, R. \& Sirot, J. (1999). Diversity of TEM mutants in Proteus mirabilis. Antimicrob Agents Chemother 43, 2671-2677.

Bradford, P. A. (2001). Extended-spectrum $\beta$-lactamases in the 21 st century: characterization, epidemiology and detection of this important resistance threat. Clin Microbiol Rev 14, 933-951.

Briñas, L., Moreno, M. A., Teshager, T., Sáenz, Y., Porrero, M. C., Dominguez, L. \& Torres, C. (2005). Monitoring and characterization of extended-spectrum $\beta$-lactamases in Escherichia coli strains from healthy and sick animals in Spain in 2003. Antimicrob Agents Chemother 49, 1262-1264.

Cantón, R. \& Coque, T. M. (2006). The CTX-M $\beta$-lactamase pandemic. Curr Opin Microbiol 9, 466-475.

Carattoli, A., García-Fernández, A., Varesi, P., Fortini, D., Gerardi, S., Penni, A., Mancini, C. \& Giordano, A. (2008). Molecular epidemiology of Escherichia coli producing extended-spectrum $\beta$-lactamases isolated in Rome, Italy. J Clin Microbiol 46, 103-108.

Chanawong, A., M'Zali, F. H., Heritage, J., Xiong, J. H. \& Hawkey, P. M. (2002). Three cefotaximases, CTX-M-9, CTX-M-13, and CTXM-14, among Enterobacteriaceae in the People's Republic of China. Antimicrob Agents Chemother 46, 630-637.

Cheng, Y. \& Chen, M. (1994). Extended-spectrum $\beta$-lactamases in clinical isolates of Enterobacter gergoviae and Escherichia coli in China. Antimicrob Agents Chemother 38, 2838-2842.

CLSI (2007). Performance Standards for Antimicrobial Susceptibility Testing, 17th Informational Supplement, M100-S17. Wayne, PA: Clinical and Laboratory Standards Institute.

Doi, Y., Adams-Haduch, J. M. \& Paterson, D. L. (2008). Escherichia coli isolate coproducing $16 \mathrm{~S}$ rRNA methylase and CTX-M-type extended-spectrum $\beta$-lactamase isolated from an outpatient in the United States. Antimicrob Agents Chemother 52, 1204-1205.

Duan, R. S., Sit, T. H. C., Wong, S. S. Y., Wong, R. C. W., Chow, K. H., Mak, G. C., Ng, L. T., Yam, W. C., Yuen, K. Y. \& Ho, P. L. (2006). Escherichia coli producing CTX-M $\beta$-lactamases in food animals in Hong Kong. Microb Drug Resist 12, 145-148.

Hirakata, Y., Matsuda, J., Miyazaki, Y., Kamihira, S., Kawakami, S., Miyazawa, Y., Ono, Y., Nakazaki, N., Hirata, Y. \& other authors (2005). Regional variation in the prevalence of extended-spectrum $\beta$ lactamase-producing clinical isolates in the Asia-Pacific region (SENTRY 1998-2002). Diagn Microbiol Infect Dis 52, 323-329.

Ho, P. L., Wong, R. C., Chow, K. H., Yip, K., Wong, S. S. \& Que, Y. L. (2008). CTX-M type $\beta$-lactamases among fetal Escherichia coli and Klebsiella pneumoniae isolates in non-hospitalized children and adults. J Microbiol Immunol Infect 41, 428-432.

Hu, G. Z., Zhang, C. H., Yuan, L., Yang, Y. R. \& Liang, J. (2005). Detection of $\beta$-lactamase and extended-spectrum $\beta$-lactamase of pathogens isolated from pig and chicken and their antibiotic susceptibility test. Agric Sci China 4, 877-882.

Hu, G. Z., Kuang, X. H., Yuan, L., Mo, J., Pan, Y. S., Fu, X. L., Xu, K. L. \& Li, L. F. (2006). Detection of antibiotic susceptibility of ESBLproducer Enterobacteriaceae against 21 agents. Zhongguo Ren Shou Gong Huan Bing Za Zhi 22, 884-887.

Hu, G. Z., Chen, H. Y., Si, H. B., Deng, L. X., Wei, Z. Y., Yuan, L. \& Kuang, X. H. (2008). Phenotypic and molecular characterization of TEM-116 extended-spectrum $\beta$-lactamase produced by a Shigella flexneri clinical isolate from chickens. FEMS Microbiol Lett 279, 162166.

Kiratisin, P., Apisarnthanarak, A., Laesripa, C. \& Saifon, P. (2008). Molecular characterization and epidemiology of extended-spectrum$\beta$-lactamase-producing Escherichia coli and Klebsiella pneumoniae isolates causing health care-associated infection in Thailand, where the CTX-M family is endemic. Antimicrob Agents Chemother 52, 2818-2824.

Liu, J. H., Wei, S. Y., Ma, J. Y., Zeng, Z. L., Lü, D. H., Yang, G. X. \& Chen, Z. L. (2007). Detection and characterisation of CTX-M and CMY-2 $\beta$-lactamases among Escherichia coli isolates from farm animals in Guangdong Province of China. Int J Antimicrob Agents 29, 576-581.

Liu, W., Chen, L., Li, H., Duan, H., Zhang, Y., Liang, X., Li, X., Zou, M., Xu, L. \& Hawkey, P. M. (2009). Novel CTX-M $\beta$-lactamase genotype 
distribution and spread into multiple species of Enterobacteriaceae in Changsha, Southern China. J Antimicrob Chemother 63, 895-900.

Livermore, D. M., Canton, R., Gniadkowski, M., Nordmann, P., Rossolini, G. M., Arlet, G., Ayala, J., Coque, T. M., Kern-Zdanowicz, I. \& other authors (2007). CTX-M: changing the face of ESBLs in Europe. J Antimicrob Chemother 59, 165-174.

Munday, C. J., Xiong, J., Li, C., Shen, D. \& Hawkey, P. M. (2004). Dissemination of CTX-M type $\beta$-lactamases in Enterobacteriaceae isolates in the People's Republic of China. Int J Antimicrob Agents 23, 175-180.

Paterson, D. L. \& Bonomo, R. A. (2005). Extended-spectrum $\beta$ lactamases: a clinical update. Clin Microbiol Rev 18, 657-686.

Pfaller, M. A. \& Segreti, J. (2006). Overview of the epidemiological profile and laboratory detection of extended-spectrum $\beta$-lactamases. Clin Infect Dis 42, S153-S163.

Ramphal, R. \& Ambrose, P. G. (2006). Extended-spectrum $\beta$ lactamases and clinical outcomes: current data. Clin Infect Dis $\mathbf{4 2}$ S164-S172.

Riaño, I., Moreno, M. A., Teshager, T., Sáenz, Y., Domínguez, L. \& Torres, C. (2006). Detection and characterization of extendedspectrum $\beta$-lactamases in Salmonella enterica strains of healthy food animals in Spain. J Antimicrob Chemother 58, 844-847.

Ryoo, N. H., Kim, E. C., Hong, S. G., Park, Y. J., Lee, K., Bae, K., Song, E. H. \& Jeong, S. H. (2005). Dissemination of SHV-12 and CTX-Mtype extended-spectrum $\beta$-lactamases among clinical isolates of Escherichia coli and Klebsiella pneumoniae and emergence of GES-3 in Korea. J Antimicrob Chemother 56, 698-702.
Smet, A., Martel, A., Persoons, D., Dewulf, J., Heyndrickx, M., Catry, B., Herman, L., Haesebrouck, F. \& Butaye, P. (2008). Diversity of extended-spectrum $\beta$-lactamases and class $\mathrm{C} \beta$-lactamases among cloacal Escherichia coli isolates in Belgian broiler farms. Antimicrob Agents Chemother 52, 1238-1243.

Song, W., Lee, H., Lee, K., Jeong, S. H., Bae, I. K., Kim, J. S. \& Kwak, H. S. (2009). CTX-M-14 and CTX-M-15 enzymes are the dominant type of extended-spectrum $\beta$-lactamase in clinical isolates of Escherichia coli from Korea. J Med Microbiol 58, 261-266.

Tenover, F. C., Mohammed, M. J., Gorton, T. S. \& Dembek, Z. F. (1999). Detection and reporting of organisms producing extendedspectrum $\beta$-lactamases: survey of laboratories in Connecticut. J Clin Microbiol 37, 4065-4070.

Wang, H., Liu, Y. M., Wang, Q. T., Sun, H. L. \& Chen, M. J. (2005). Identification of DHA-1 plasmid-mediated AmpC and CTX-M extended-spectrum- $\beta$-lactamase-producing Klebsiella pneumoniae in Beijing. Zhonghua Wei Sheng Wu Xue He Mian Yi Xue Za Zhi 25, 419422.

Woodford, N., Ward, M. E., Kaufmann, M. E., Turton, J., Fagan, E. J., James, D., Johnson, A. P., Pike, R., Warner, M. \& other authors (2004). Community and hospital spread of Escherichia coli producing CTX-M extended-spectrum $\beta$-lactamases in the UK. J Antimicrob Chemother 54, 735-743.

Xiao, Q. Z., Su, D. H., Jiang, J. H. \& Zhong, N. S. (2008). Genotypes of CTX-M-like-extended-spectrum and OXA-like-spectrum $\beta$-lactamase in clinical isolates of Gram-negative bacilli in Guangzhou. Zhonghua Yi Yuan Gan Ran Xue Za Zhi 15, 1321-1326. 